\title{
Study on Solution and Application of Virtual Park Zone Network
}

\author{
Dingfu Luo \\ Computer Department \\ Guangdong Songshan Polytechnic College \\ Shaoguan, Guangdong, China 512126
}

\author{
Haijun Qian \\ Adult Continuing Education Department \\ Zhu Hai City Polytechnic \\ Zhuhai, Guangdong, China 519000
}

\begin{abstract}
Putting forward the model to deploy virtual park zone basing on the background of new technology such as the Cloud Computing and the Internet of things, to compare and discuss the drawbacks of traditional network architecture from the virtualization and multiple service bearer, to give a method of horizontal integration to simplify and expand network flexibly, and of multiple service bearer, finally to design a solution of application of virtual park zone network.
\end{abstract}

Keywords-virtual park zone; virtualization; multiple service bearer; horizontal integration; solution

\section{INTRODUCTION}

Cloud computing distributes powerful computing capacity onto clients with the help of advanced commercial models like SaaS, IaaS, and PaaS [1]. Compare the newly released series of software products with original products of the big manufacturers, there are mainly two big changes: one is enterprise coordination: it allows multiple persons to process one document at the same time, which changes the serial business processing way; the other is access according to demand: user may use products that could only be used on computers traditionally by computer, cell phone or mobile terminal. The future application development tendency can be seen from such representative changes. Therefore, when the upper layer application architecture of the enterprise starts transformation from fixed terminals to diversified terminal, from fixed space to flexible space, from low-efficient serial processing to high-efficient real-time concurrent processing, how should we construct the network architecture? And how should we deploy network authentication way, access way, convergence design, real-time? The practical application development forces us to think further. With the proposing of concepts of Smarter Planet and Internet of Things, as well as their application in fields of logistics, electric power, and security guard, the access information point of network is no longer only human, but referring to wider objects, such as sensor, camera, laser scanner, etc. All these are the objects to be accessed to the future network. Internet of Things proposes new requirements to network: for example: object identifier: "under the situation that the IPv4 address resources have been used up, how to adopt unique identifier to identify numerous object?" Taking application interoperability for instance, "Lots of single business systems have emerged, like smart grid and logistics positioning. However, the charm of Internet of Things

Fund Project: Higher Vocational and Technical Education Research Association of Guangdong Province (GDGZ15Y119) lies in its capacity of jointing different systems to create amazing effect, like jointing to food safety system and pollution source monitoring system." This would surely result in heterogeneous network fusion, application front-end processing, system isolation and interoperability. This proposes new demands to network architecture. It can be seen from the above two aspects that, no matter the boundless cloud computing network, or the Internet of Things which develops access objects from human to things, proposes tough challenge to traditional network architecture.

\section{DisADVANTAGES OF TRADITIONAL NETWORK ARCHITECTURE}

\section{A. Spatial Limit}

Traditional network architecture is usually designed under the precondition that specific person adopts specific equipment in specific space. The adopted technologies rarely consider the mobility of human, or even for consideration of safety, adopting binding technology. Though it settles some certain problems to certain extent, it sacrifices flexibility and loses the business expansion capacity.

\section{B. Complicated Structure}

The traditional network architecture is becoming more and more complicated. The 10 gigabit core layer, high-intensity business processing convergence layer, and low-end tiny access layer are all of values of existence. Since the currently existing network architecture exposes details on upper application layer, which makes lots of application deployments become hard to implement due to the disadvantage that the network can not screen technical details properly.

\section{Lack of Unity}

Due to the difference of access modes and objects, customized demands as safe and wireless access become more and more. However, new demands haven't been totally fused into the network and result in lots of separation problems.

\section{SOlUtion OF VIRTUAL PARK ZONE NETWORK}

The best way for engagement of business is to ignore the bottom infrastructural facility difference and deploy in mode of loose coupling. For current and future park zone network design, it is the main thinking point of how to make the 
network access mode, flow rate planning, high usability design, and safety strategy look like transparent air to the business and make the park zone network become real carrier platform for elastic business extension. Therefore, the fundamental target of the current new solution is to make the network more simple and transparent. For "simple", it not only refers to the simple network architecture, but also refers to the simple integrated running and maintaining process for new business support and organization architecture adjustment; and for "transparent", it not only refers to less adopted network protocols, but also effectively simplified network structure to screen the bottom layer difference.

For such consideration, deployment of virtualization and multi-business carrying technical solution in park zone network has become the focus[3]. Virtualization is represented in horizontal and vertical isolation of network, hoping to make the network a pool-based resource; multi-business carrying is represented in network difference in terms of customized user demands, such as screening of access space, measure, and safety demand, hoping to make the network interface easier and enhance user experience. Besides vertical isolation virtualization deployment, horizontal integration is also adopted to reach the purposes of simplifying and flexibly extent the network, and make the modularized products gradually become the resources that can be shared in the virtual group by virtualization technology, thus to realize further transmission from modularization to resource.

\section{A. Adopt Horizontal Integration to Simplify Network}

Network simplification can be analyzed from two aspects: simplify park zone network topology and simplify park zone network protocol deployment.

As shown in "Fig. 1", in such classical park zone network design, it adopts multi-layer structure according to access, convergence, and core: in order for the convenience of user, it commonly designs access layer network as 2-layer access, and sets the 3-layer gateway of the client at the convergence layer equipment; meanwhile, in order to guarantee the HA capacity of the gateway [4], the convergence layer is adopted with dualnode redundancy network; core layer is also adopted with dual-node network to enhance performance and redundancy capacity. However, such traditional park zone network structure has gradually become hard to satisfy requirements of new services: 2-layer access network generates multiple loops between access layer and convergence layer. Such loops form looped network. And the ever adjusting IT business of the enterprises further makes the looped network scale be enlarged or complicated; part of the network eliminate loops in order to reduce complexity. However, such way brings low usability problems as single link and single-point access. Therefore, it is in urgent demand to eliminate loop and simplify network topology while guaranteeing multi-business, flexibility, reliability, easy-to-use, and extensibility performance.

Virtual park zone network solution solves the above problem by bringing IRF2 technology [5] into park zone network solution.

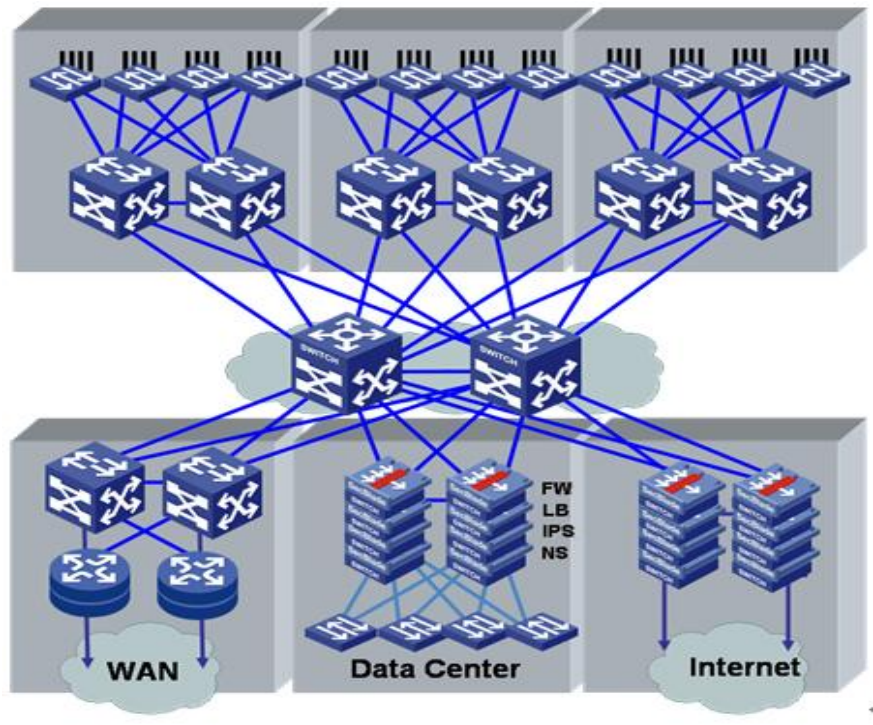

Fig. 1. Traditional Park Zone Structure

As shown in "Fig. 2", in the enterprise park zone network architecture, the IRF2 technology is adopted to perform horizontal integration respectively in network convergence and core layers, virtualize multiple redundancy equipment into single logic equipment, and form a network management and forwarding node; it performs IRF2 integration in complicated access environment of access layer, virtualize 9 physical network nodes as single unit equipment, totally eliminate access layer loop, and form bundle link high bandwidth and reliability upper link. Under such virtualization, netted enterprise park zone network can form a very simple architecture with all layers linking by bundle single logic links, eliminating loop. It no longer needs to design complicated spanning tree protocol in the access layer, and no longer needs to run VRRP protocol [6] on client access gateway which needs to turn to be single logic node.

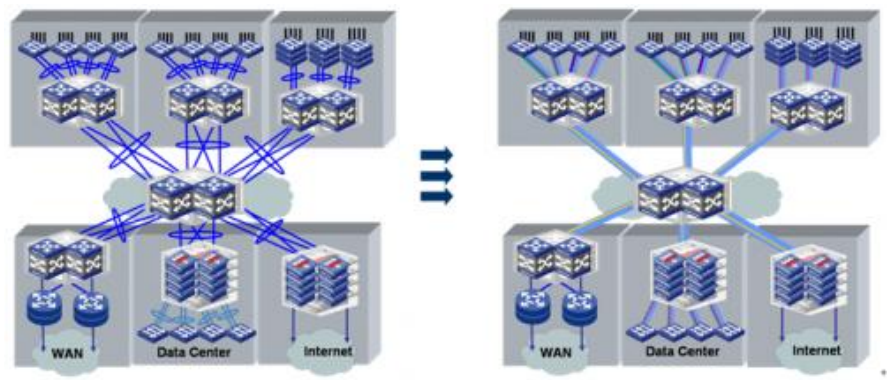

Fig. 2. End-to-end Enterprise Network IRF2 Architecture

End-to-end IRF2 deployment realizes no-loop, tree-type, and radiation network topology structure of the park zone, which greatly simplifies the running maintenance and management work. Macro path of the data flow in the network is accorded with the simplified integrated network topology. The business flow direction in the network is clear. Meanwhile, the expansion of each IRF2 node itself (such as add device to this node) would not change the logic structure of the enterprise network or affect the protocol interaction between 
upper and lower levels of networks. Such virtual network represents optimized integrated architecture. On one hand, it can simplify the park zone network protocol deployment. It turns the loop network to be tree-type network by eliminating the access and gathered loops in the network, which makes the netted enterprise park zone network form a very simple architecture. And in the architecture, all layers link each other via bundle single logic link, eliminating loop. It no longer needs to design complicated spanning tree protocol in the access layer, and no longer needs to run VRRP protocol on client access gateway which needs to turn to be single logic node. And on the other hand, when traditional park zone scale is developed to reach certain degree, all parts of the network would form netted network structure. Therefore, the router of the whole network is designed according to the region-based division. Taking OSPE [7] for instance, commonly, the network core and key network components are set at area 0 while the network sub-modules are set at area 1, 2, 3. After taking IRF2 for performing end-to-end virtualization, the network structure becomes clearer, and the router structure of the whole network is also simplified. Regional router forms point-to-point and point-to-multipoint simple structures due to the simplification of logic links, which greatly reduces router quantity relating to network devices, so as the router computing volume of the whole network. Meanwhile, link bundle along network layers avoids affecting of single physical link fault to upper layer router. This makes the end-to-end IRF2 network architecture have a stable simplified router structure. The expansion of network scale would not intensify the complexity of routers. And such kind of router structure not only simplifies the network router design, reduce device requirements, reduce device load, but is also helpful for enterprise network long-term planning and modularized expansion.

In a word, it adopts horizontal integration technology, which not only simplifies protocol deployment, but also guarantees the 2-layer expansion of network, not affecting logic topology and router status of the network. It realizes screening of 2-layer expansion for network management, which better satisfies the park zone network demands of simple deployment, low maintenance cost, and free expansion.

\section{B. Flexible Extension of Horizontal Integration}

Extensibility is usually difficult in network access layer design. Due to the organization development, enterprises have demand on IT fundamental rapid development. In terms of large scale modularized network extension mode, traditional core network structure is able to support in proper way. But in access layer, it usually faces the difficulty of hardly satisfying requirement of extensibility, because the access layer network topology is complicated (it usually is 2-layer access mode), and the extension of equipment and port may further worsen the complexity of the network structure. Targeting for the large scale increase of user terminals, IRF2 corresponding plan is: add members in IRF2 system of access layer to perform port extension and satisfy the ever growing access ports requirement. The extended system would have no affect on other part of the network and form smooth extensibility. If having higher requirement on upstream bandwidth, it can smoothen and upgrade bandwidth by adding IRF2 system upstream aggregation link members.

\section{Multi-business Carrying}

Access environment deployed with IRF2 system could provide rich network service design to provide better support to business deployment. Equipment under IRF2 architecture could support H3C EAD endpoint access safety solution, provide enterprise park zone overall safety access capacity, and solve the inside-park-zone mobile office application problem based on identification network access and dynamic strategy; PoE[8] function has been successfully brought in with basic functions of exchanger. And with the continuous launching of new technology standards, the following IRF2 would support intermediate and high power PoE standards, thus to satisfy new power supply business demands of enterprise network, such as cctv thin terminal (such as PoE current-carrying terminal), video telephone, voice telephone, wireless AP, etc,; the integrated Voice VLAN[9] function provides convenience for the AP voice accessed to IRF2. Meanwhile, by deploying MPLS VPN[10], it settles the overlapped IP problem under condition of sharing a one physical network environment at the time of multi-network fusion. Besides, it establishes end-to-end business channels for various applications and avoids the problem of $\mathrm{CE}$ dual upstream planning problem under circumstance of dual PE (Concept in MPLS VPN) by deploying IRF2[11]; multicast VPN network service deployment settles the problems of monitoring business melting in park zone network and the following $\mathrm{OA}$ interaction.

Therefore, the virtual park zone network solution forms good architecture to support OA, multimedia business, monitoring network, and cross-department coordination by VPN, Safety, Wireless, Multicast, and Voice network services during the development process of transforming from park zone network to resource-based exchange by IRF2 horizontal integration and VPN vertical isolation technology.

\section{Integrated Deployment of Virtual Park Zone Network}

After integrating the horizontal virtualization and vertical virtualization technologies, the integrated deployment of virtual park zone network is as shown in "Fig. 3". IRF2 technology is adopted to perform horizontal integration in access layer, convergence layer, and core layer. After integration, the safety module could be deployed on the exchanger of convergence layer, front-end exchanger of DataCenter, and exchanger of outlet front-end of Internet flexibly in form of board card. 


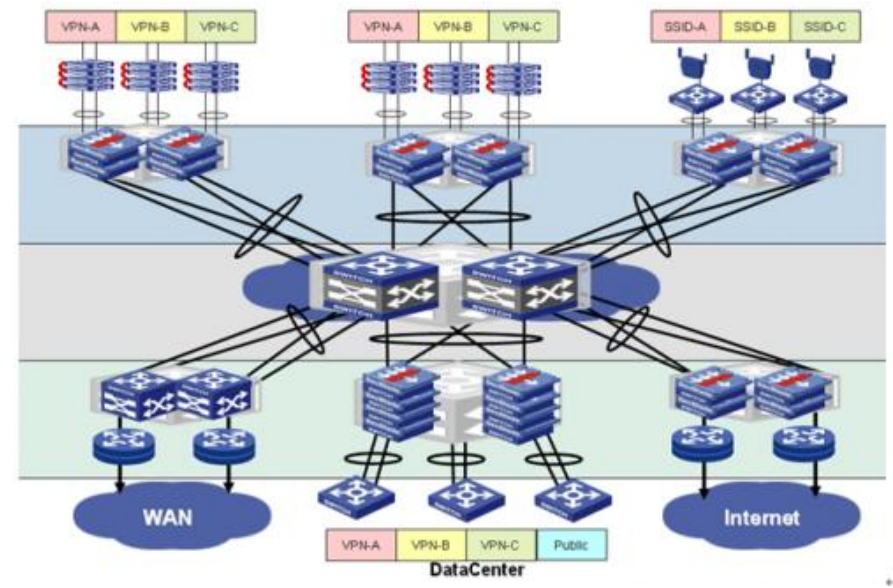

Fig. 3. Integrated Deployment of Virtual Park Zone Network

The whole network performs path virtualization by MPLS/VPN or MCE multi-hop technology to realize isolation to network resource. It accomplishes integrated virtual park zone network architecture by deploying horizontal virtualization and vertical virtualization technologies.

\section{BeSt PRACTICE OF VIRTUAL PARK ZONE NETWORK}

\section{A. Best Practice of Government Affairs Administration Center}

A new administration center park zone of a certain city is composed of tens of buildings. When the park zone construction is finished, there will be over a hundred of governmental departments moving in. And the network would be responsible for various e-governmental affairs application. The informatization construction of this park zone not only requires providing a unified platform environment, but also requires accomplishing integration and development of egovernmental affairs. The construction of this park zone is of great significance and difficulty. It shall pay great attention on aspects of reliability and extensibility.

- Network management and maintenance are hard to implement because it contains tens of thousands of information points and hundreds of units of equipment;

- The network is performed with redundancy deployment in order to guarantee reliability. But this causes too many network loops. And VRRP+MSTP is needed to be deployed to avoid broadcast storm;

- Too much wireless and safety demands, especially for Internet export, so safety shall be the key point to be considered;

- For the over a hundred of governmental departments, they have both independent business demand and sharing business demand. Therefore, during the egovernmental affairs integration, the network shall be featured in flexible performance to adapt to the business adjustment.
By deploying virtual park zone network, the topological structure as shown in "Fig. 4" would be formed to satisfy customers' demand: [14].

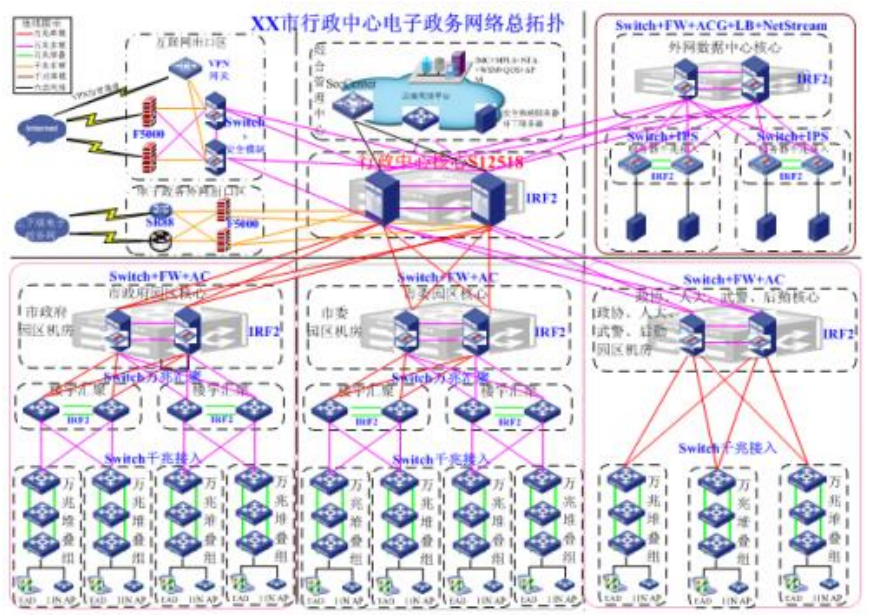

Fig. 4. Best Practice of Government Affairs Center

- The whole network is performed with horizontal integration and reduces network management and forwarding nodes to $1 / 6$ of original by equipment virtualization of core, convergence, and access, thus to reduce the management and maintenance difficulty.

- It provides seamless extension capacity, and guarantees no logic topology change would happen when performing access, convergence, and core equipment horizontal extension, thus reach smooth extension.

- Eliminate network loop: it eliminates the loops in the redundancy network, avoids VRRP+MSTP protocol deployment, and provides faster convergence speed;

- It reduces router processing in the network, reduces quantity of routing neighbors in the network, thus to further reduce router quantity of current network to be less than half o the traditional solution.

- Integrated cabled and wireless network design, integrated network safety design, and modularized deployment under virtualized environment guarantee the realization of resource-based deployment of network businesses.

- The vertical isolation MPLS VPN deployment not only satisfies the business isolation and sharing access demands, but also provide good extensibility to future business development.

Therefore, it settles the business demand of the administration center of a certain city by deploying virtual park zone network solution, and proposes network architecture that is more transparent than traditional network architecture and network solution that is easier for extension.

\section{B. Best Practice of Enterprise Park Zone}

A certain newly built factory is of clear business types. But with the development of business, it proposes lots of 
requirements on network architecture of the newly built park zone:

- Businesses are divided into office, production, and monitoring, which need business isolation;

- MIS needs interaction of monitoring network and office system, so that it needs interaction on businesses;

- It has high requirements on wireless and safety, which need integrated consideration.

- In order to guarantee reliability, it needs redundancy deployment on the whole network, which makes lots of network loops exist in the network.

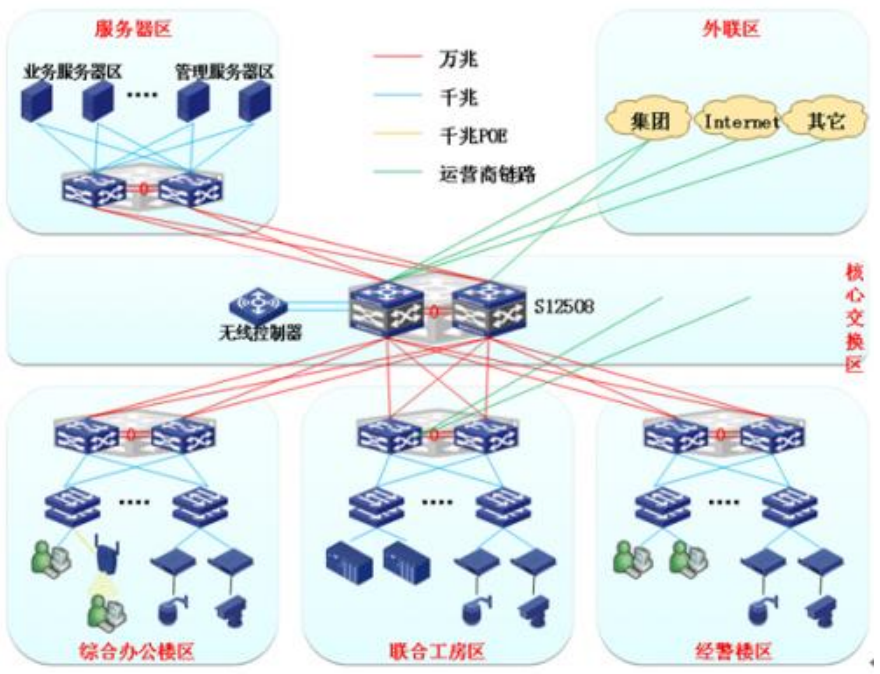

Fig. 5. Best Practice of Enterprise Park Zone

It performs deployment based on virtual park zone network solution to satisfy customer demand on network:

- Adopt MPLS VPN to perform vertical partition for network thus to guarantee logic isolation of office, production, and monitoring businesses;

- Satisfy office and monitoring businesses mutual access via sharing VPN technology.

- Make integrated design for cabled and wireless, network and safety, and manage via unified management platform;

- Eliminate network loop via horizontal equipment integration, thus to avoid deployment of VRRP+MSTP protocol and reduce the router quantity in the network and enhance the network convergence speed.

It settles the enterprise park zone business isolation and mutual access demands of the enterprise park zone via the deployment of virtual park zone network solution, and provides network solution with stable logic architecture to satisfy the current and future business network demands, thus to provide good support to the informatization construction of the enterprise park zone.

\section{CONCLUSION}

As the next generation enterprise park zone network construction thought, park zone virtualization solution provides a complete set of ways realizing virtualization. It integrates equipment and link resources on equipment layer and integrates path and safety service resources on logic layer, thus to enhance the management, reliability and performance of the network via horizontal virtualization technology, and realize terminal access safety and access privilege control, safe isolation of business data transmission, distribution of application resources according to demand, concentrated network administration, and strategic deployment by vertical virtualization technology.

The enterprise IT business has been becoming more and more complicated and important. As the carrier of such key business, the enterprise network shall be able to adapt to the changes to satisfy the enterprise business development. It can be forecasted that, through further integration and virtualization to enterprise network resource, the next generation of park zone network would screen mature technologies in the bottom layer by virtualization technology, which would make the logic simpler and integrate the park zone network as a matrix, for which, the network resources and safety resources can be dynamically divided and carried out with logic isolation, thus to provide more powerful business carrying capacity and continuously satisfy enterprise's demand on IT development.

\section{REFERENCES}

[1] Wikipedia Cloud computing [EB / OL] .http: //en.wikipedia.org/wiki/Cloud_computing,2009.

[2] Zhu Zhongying. The Progress and Trends of Sensor Networks and Internet of Things [J]. Microcomputer Applications, 2010,26 (1): 13.DOI: 10.3969 / j.issn.1007-757X.2010.01.001.

[3] Zeng Weimin. 75 Servers Reduced into Six [C].// 2012 (The Third) Symposium of China Communications Industry Cloud Computing Summit .2012: 101-103.

[4] Su Xue. Design and implementation of DFT/ HA Signaling Protocol Stack of Softswitch-Based Signaling Gateway[D]. East China University of Technology, 2004.

[5] Sun Zhenhua, Zhao Mi, Sun Nannan et al. On the Application of IRF2 and Campus Network [J]. Journal of Shandong Vocational and Technical College of Business, 2012,12 (2): 95-98.DOI: 10.3969 / j.issn. 1671-4385.2012.02.029.

[6] Luo Dingfu, Chen Yongsong. Design and Implementation of VRRP Multiple Backup Group [J]. journal of Qiqihar University: Natural Science Edition, 2012,28 (4): 70-72.DOI: 10.3969 / j.issn.1007-984X. 2012.04.023.

[7] Dan-Dan Wang, Li-Gang Dong, Fu-Rong Zhou et al. Study of OSPF Routing Optimization among Forwarding Elements in the ForCES Router [J] .Information Technology Journal, 2012,12 (2).

[8] Liu Kaiju. Research and Implementation of POE Function of Ethernet Switch [D] Beijing Jiaotong University, 2006.DOI: 10.7666 / d.y1081413.

[9] Zhang Peng. Voice VLAN Design Based on Layer3 Lan Switch Protocol Stack [J]. Chinese New Telecommunications, 2013, (21):. 9798.DOI: 10.3969 / j.issn.1673-4866.2013.21.070.

[10] Jiang Dongyi, Lv Shuwang, Luo Xiaoguang et. al. Analysis on Key Technologies of VPN [J]. Computer Engineering and Applications, 2003,39 (15):. 173-177.DOI: 10.3321 / j.issn: 1002-8331.2003.15.056. 
[11] Hou Jianfeng, Ma Mingkai. Study on PE-CE Interconnect Simulation in MPLS VPN [J]. Computer Engineering, 2010,36 (12): 123-125.DOI: 10.3969 / j.issn.1000-3428.2010.12.042.

[12] http://www.h3c.com.cn/Solution/Base_Network_Secrity/Dummy_Gard en_Solutions/Garden_Dummy_Solution/What_Do_I_Need/Bookmark/2 00805/606722_30004_0.htm.

[13] Luo Dingfu, Li Xialong. Design and Implementation of Load Balancing Experiment of Multiple Spanning Tree Protocol [J] Computer Development and Applications, 2013, (10): 39-41.DOI: 10.3969 / j.issn.1003- 5850.2013.10.013.

[14] Luo Dingfu, Li Yan. Technological Research on Connection of Layer3 Lan Switch and Routers[J]. Journal of Foshan University (Natural Science), 2013,31 (2): 52-54.DOI: 10.3969 / j.issn .10080171.2013.02.011.

[15] Yao Xiushan. 3-tier Logical Architecture Methods of Network Exit[J]. Chinese Education Informatization - Higher Vocational Education, 2010, (4): 26-27.DOI: 10.3969 / j.issn.1673-8454.2010.04.010. 